\title{
Phosphoinositide 3-kinase/protein kinase B regulates inflammation severity via signaling of Toll-like receptor 4 in severe acute pancreatitis
}

\author{
JING WANG ${ }^{1,2}$, CHUN ZHANG $^{1}$, PING XU ${ }^{1,2}$, ZHI-WEN YANG $^{3}$, CHENG-ZHAO WENG $^{4}$ and YUE-XING LAI ${ }^{1,2}$ \\ ${ }^{1}$ Department of Gastroenterology, Shanghai Songjiang Hospital Affiliated to Nanjing Medical University, Shanghai 201600; \\ Departments of ${ }^{2}$ Gastroenterology and ${ }^{3}$ Pharmacy, Songjiang Hospital Affiliated Shanghai First People's Hospital, \\ Shanghai Jiao Tong University, Shanghai 201600; ${ }^{4}$ Department of Gastroenterology, \\ Zhongshan Hospital, Fudan University, Shanghai 200030, P.R. China
}

Received March 26, 2017; Accepted March 21, 2018

DOI: $10.3892 / \mathrm{mmr} .2018 .8819$

\begin{abstract}
Phosphatidylinositol 3-kinase (PI3K)/protein kinase B (Akt) has been indicated to serve an important role in the pathogenesis of inflammatory diseases. It was previously demonstrated that the PI3K/Akt inhibitor wortmannin alleviated the severity of inflammation and improved the survival rate in rats with induced severe acute pancreatitis (SAP), which indicates that PI3K/Akt may serve a role in the pathogenesis of acute pancreatitis. To date, the mechanism by which PI3K/Akt regulates inflammation has not been elucidated. In the present study, it was hypothesized that PI3K/Akt may be invovled in SAP inflammation via regulation of the Toll-like receptor 4 (TLR4) signaling pathway. Rats with SAP were treated with the PI3K/Akt agonist insulin-like growth factor (IGF)-1, which alleviated the severity of inflammation in a dose-dependent manner. Furthermore, to better understand the role of PI3K/Akt in inflammation, RAW264.7 murine macrophages were stimulated with IGF-1 and wortmannin alone or together before the induction of inflammation by treatment with lipopolysaccharide (LPS). The results indicated that LPS stimulated overexpression of TLR4, myeloid differentiation primary response gene 88 (MyD88), PI3K, Akt, p38MAPK and NF-кBp65 mRNA, and increased the levels of tumor necrosis factor (TNF)- $\alpha$ and interleukin (IL)- 6 in RAW264.7 cells compared with the control group. The levels
\end{abstract}

Correspondence to: Dr Ping Xu, Department of Gastroenterology, Shanghai Songjiang Hospital Affiliated to Nanjing Medical University, 746 Middle Zhongshan Road, Shanghai 201600, P.R. China

E-mail: sjzxxp@yeah.net

Abbreviations: PI3K/Akt, phosphoinositide 3-kinase/protein kinase B; TLR4, Toll-like receptor 4; SAP, severe acute pancreatitis; TNF- $\alpha$, tumor necrosis factor- $\alpha$, IL- 6 , interleukin- 6

Key words: phosphoinositide 3-kinase/protein kinase B, toll-like receptor 4 , severe acute pancreatitis of all detected factors were increased by stimulation with IGF-1, whereas these levels were decreased following treatment with wortmannin alone, and the effect of IGF-1 was abolished by wortmannin in RAW264.7 cells. In vivo studies indicated that IGF-1 produced the same anti-inflammatory effect as wortmannin and that expression of TLR4, p38MAPK and NF- $\kappa$ Bp65 decreased following treatment with IGF-1. These findings indicate that PI3K/Akt may take part in the progression of SAP by regulating the TLR4 signaling pathway and that IGF-1 can inhibit inflammation in SAP rats.

\section{Introduction}

Severe acute pancreatitis (SAP) is an inflammatory disease of the pancreas characterized by elevated pancreatic enzymes in the blood which can lead to multiple organ dysfunction and is associated with high mortality rates (1). SAP involves a complex cascade of events. The progression of SAP leads to acinar cell injury, and as a result of inflammation and tissue damage, inflammatory cells including macrophages, neutrophils and dendritic cells are actively recruited to the diseased site and induce the rapid production and release of inflammatory cytokines such as tumor necrosis factor (TNF)- $\alpha$ and interleukin (IL)-6, which play the dominant role in local pancreatic inflammation and systemic complications. Our previous research showed that the phosphatidylinositol 3-kinase (PI3K)/protein kinase B (Akt) inhibitor wortmannin could decrease the production of inflammatory cytokines, alleviate the severity of inflammation in the pancreas and improve the survival rate in SAP rats, indicating that the PI3K/Akt signaling pathway is involved in the development of SAP and the severity of inflammation through the regulation of inflammatory cytokines (2). However, the mechanism of PI3K/Akt in SAP is not fully understood.

One Study confirmed that Toll-like receptors (TLRs) and PI3K interact to modulate inflammation (3) TLRs are the first-line of host defense guards that sense invading microbes to induce innate and adaptive immune responses. Since the activation of downstream signaling pathways culminates in the production of proinflammatory mediators, TLRs are closely involved in the developmental stages of a variety of 
inflammatory diseases, include SAP (4-7). TLR4 activation by lipopolysaccharide (LPS) triggers the association of myeloid differentiation primary response gene 88 (MyD88) with the cytoplasmic domain of TLR4, which recruits IL-1 receptor-associated kinase 4 (IRAK4) and sequentially phosphorylates IRAK-1, activating a series of signaling molecules, kinases, transcription factors and inflammatory genes $(8,9)$. NF- $\mathrm{BB}$ and p38MAPK are two major downstream signaling pathways in the TLR4 signaling pathway $(9,10)$. It is well known that $\mathrm{NF}-\kappa \mathrm{B}$ and $\mathrm{p} 38 \mathrm{MAPK}$ play critical roles in regulating the expression of proinflammatory genes such as TNF- $\alpha$, IL- $\beta$ and IL-6 $(11,12)$. Our previous findings indicated that these two signaling pathways were activated in SAP rats $(2,13)$ and pancreatitis-associated ascitic fluid stimulated THP-1 cells (14), which was suppressed by wortmannin (2). In the present study, we examined the expression of $T L R 4, P I 3 K$, Akt, p38MAPK, NF- $\kappa$ Bp 65 and $M y D 88$ in vivo in SAP rats and in vitro in RAW264.7 murine macrophages (stimulated by LPS) following treatment with the PI3K agonist insulin-like growth factor (IGF)-1, and further explored the possible mechanisms by evaluating the correlation between PI3K/Akt and TLR4 in inflammatory reactions associated with SAP. Our results demonstrate for the first time to our knowledge evidence that PI3K/Akt may regulate the TLR4 signaling pathway and take part in the progression of the SAP inflammation reaction.

\section{Materials and methods}

Animals and treatments. Thirty healthy male Sprague-Dawley rats weighing 230-270 g were purchased from the Animal Center of Shanghai Jiao Tong University (Shanghai, China). Animals were housed in cages under a controlled temperature of $22 \pm 1^{\circ} \mathrm{C}$ in 12 -h light-dark cycles, were fed standard laboratory chow and provided water ad libitum and allowed to acclimatize for at least 1 week. The experiment was designed in accordance with the guidelines for the care and use of laboratory animals in research and was approved by the Ethical and Research Committee of Shanghai Jiao Tong University. Sprague-Dawley rats were randomly divided into five groups: Normal group (Normal), sham operation group (SO), SAP model group (SAP), high-dose IGF-1 treatment group (High-dose IGF-1) and low-dose IGF-1 treatment group (Low-dose IGF-1), n=6 per group. Surgical anesthesia was accomplished by intraperitoneal injection of $2 \%$ pentobarbital sodium ( $40 \mathrm{mg} / \mathrm{kg}$ bw). SAP was induced by retrograde infusion of $5.0 \%$ sodium taurocholate ( $1 \mathrm{ml} / \mathrm{kg}$ body weight) into the pancreatic and biliary duct. Half an hour before inducing SAP, rats were injected intraperitoneally with 20 or $200 \mu \mathrm{g} / \mathrm{kg}$ of IGF-1 (Sigma-Aldrich, USA) in the low- and high-dose IGF-1 groups, respectively. Rats in the sham group underwent a sham operation with nothing infused. All animals were anaesthetized by $2 \%$ pentobarbital sodium (50 mg/kg bw) prior to sacrifice and sacrificed by cervical dislocation. Blood, ascitic fluid and pancreatic tissue samples were obtained $6 \mathrm{~h}$ after ductal infusion of sodium taurocholate. Arterial blood samples were obtained from the abdominal aorta. Blood samples were centrifuged at 3,000 g for $10 \mathrm{~min}$ at $5^{\circ} \mathrm{C}$ and the supernatants were separated with sterile pipettes and frozen at $-80^{\circ} \mathrm{C}$ for the following assays. Before the rats were sacrificed, the sutured abdominal incisions were opened. Changing the rat's position allowed the ascites to accumulate, which was then collected by $10 \mathrm{ml}$ syringe. The maximum volume of ascites observed in the study was $5.06 \%$ (i.e., $12 \mathrm{ml}$ in a rat of $235 \mathrm{~g}$. These $12 \mathrm{ml}$ of ascites weighted $11.89 \mathrm{~g}$ ). Ascites fluid was stored at $-80^{\circ} \mathrm{C}$ until assay. A portion of the pancreatic tissue samples was immediately frozen and maintained at $-80^{\circ} \mathrm{C}$ for real-time polymerase chain reaction (PCR), and the remaining tissue was fixed in $10 \%$ formaldehyde for histopathological examination. Pathological examination was as follows: The pancreatic tissue from each rat was collected and fixed in 10\% neutral-buffered formaldehyde, embedded in paraffin and stained with hematoxylin and eosin. Edema, inflammation, as well as hemorrhage and necrosis of the pancreas were each graded from 0 to 4 as described below: Edema (0, no edema; 1, focally increased between lobules; 2, diffusely increased between lobules; 3 , tense acini, widely separated lobules; and 4, gross lobular separation); inflammation (0, no leukocytes; 1, 2-10 leukocytes/high-power magnification [HP]; 2, 11-20 leukocytes/HP; 3, 21-30 leukocytes/HP; and 4, $>30$ leukocytes/HP); hemorrhage ( 0 , no hemorrhage; 1 , blood in $25 \%$ of parenchyma; 2 , blood in $25-50 \%$ of parenchyma; 3 , blood in $50-75 \%$ of parenchyma; and 4 , blood in $100 \%$ of lobules); necrosis ( 0 , no necrosis; 1 , periductal parenchymal destruction; 2, focal parenchymal necrosis; 3, diffuse loss of lobules; and 4, severe loss of lobules). The pathological scores in the study were presented as the sum of these different scores.

Cell culture and treatment. RAW264.7 murine macrophages from the Chinese Academy of Science Cell Bank were grown in RPMI 1640 supplemented with $10 \%$ fetal bovine serum at $37^{\circ} \mathrm{C}$ in a $5 \% \mathrm{CO}_{2} /$ air environment. The cells were resuspended at a concentration of $5 \times 10^{5}$ cells $/ \mathrm{ml}$ in culture medium. A 1-ml cell suspension was transferred to a six-well plate and incubated with a final concentration of $1 \mu \mathrm{g} / \mathrm{ml}$ LPS (Sigma-Aldrich; Merck KGaA, Darmstadt, Germany). Cells were divided into five groups: Blank group, LPS group, IGF-1 group [cells were incubated with IGF-1 (100 ng/ml) $1 \mathrm{~h}$ before treatment with LPS], wortmannin group [cells were incubated with wortmannin (100 nM; Sigma-Aldrich; Merck KGaA) 30 min before treatment with LPS] and IGF-1+wortmannin group (cells were incubated with both IGF-1 (100 ng/ml) and wortmannin (100 nM) before treatment with LPS). The Blank group was incubated in culture medium without drugs. The reaction was stopped after a $6-\mathrm{h}$ incubation. After centrifugation $(1,000 \mathrm{xg}, 6 \mathrm{~min})$, the supernatant and cell lysates were collected and stored at $-80^{\circ} \mathrm{C}$ for subsequent analysis.

Cell viability assay. We used a Cell Counting Kit-8 (CCK-8) assay (Dojindo Molecular Technologies, Inc., Kumamoto, Japan) to evaluate cell viability. Cells were collected in the logarithmic growth phase and plated at a density of $1 \times 10^{3}-5 \times 10^{3}$ cells per well in 96 -well plates in triplicate, incubated at $37^{\circ} \mathrm{C}$ for $24 \mathrm{~h}$, then treated with increasing concentrations of LPS $(0,0.1,0.3,1,3$ and $10 \mu \mathrm{g} / \mathrm{ml})$, IGF-1 $(0,1,10,30,100$ and $300 \mathrm{ng} / \mathrm{ml})$ and wortmannin $(0,3,10,20$, 100 and $300 \mathrm{nM}$ ), while the Blank group was incubated with culture medium without drugs. After $24 \mathrm{~h}$ incubation at $37^{\circ} \mathrm{C}$, every well was incubated with CCK- 8 reagent in complete medium at a ratio of 1:10 for $1 \mathrm{~h}$. The absorbance value of each well was measured at $450 \mathrm{~nm}$ using a microplate reader (Molecular Devices, LLC, Sunnyvale, CA, USA). 
Histopathological examination. At $6 \mathrm{~h}$ after inducing SAP, pancreatic tissue was removed and fixed in $10 \%$ neutral-buffered formalin. Samples were embedded in paraffin, sliced into 4- $\mu \mathrm{m}$ sections and stained with hematoxylin-eosin in preparation for blinded histologic assessment. Histologic changes were evaluated in random, nonconsecutive fields (Olympus BX51/Olympus DP71; Olympus Corporation, Tokyo, Japan). Edema, inflammation, as well as hemorrhage and necrosis of the pancreas were each graded from 0 to 4 by the standard of Schmidt et al (15), and the total score was obtained by addition of the four aspect scores.

Ascite amylase activity and ascite volume measurement. The amylase activity in samples of ascites fluid was measured with an automated biochemistry analyzer. Ascite volume was also measured.

Expression of TNF- $\alpha$ and IL- 6 assay. IL-6 levels in sera and TNF- $\alpha$ and IL- 6 levels in cell supernatants were assayed with ELISA kits (Nanjing Jiancheng Bioengineering Institute, Nanjing, China). ELISA was performed according to the manufacturer's instructions.

Quantitative PCR ( $q P C R)$. qPCR was used to investigate the expression of TLR4, IGF-1 receptor (IGF-1R), p38MAPK and NF- $\mathrm{Bp} 65 \mathrm{mRNA}$ in pancreatic tissue, and expression of PI3K, Akt, TLR4, MyD88, p38MAPK and NF- $\kappa$ Bp65 mRNA in RAW264.7 murine macrophages. Total RNA was prepared from tissue and cells using RNAiso (Takara Bio, Inc., Otsu, Japan) according to the manufacturer's protocol. Reverse transcription of total RNA was performed for synthesis of cDNA using a Takara RNA PCR kit. qPCR was performed using the Light Cycler Nano instrument (Roche Diagnostics, Indianapolis, IN, USA). Calculations were performed using $G A P D H$ as an internal reference gene. The gene-specific primers used in pancreatic tissue were as follows. TLR4: Sense 5'-ATGCTAAGGTTGGCACTCTC-3', antisense 5'-CAGGCA GGAAAGGAACAATG-3'; $N F-\kappa B p 65$ : Sense 5'-AGACCT GGAGCAAGCCATTAG-3', antisense 5'-CGGACCGCATTC AAGTCATAG-3'; p38MAPK: Sense 5'-TTCCCAGCAGTC CTATCC-3', antisense 5'-CAGATGGCAAGGGTTCAG-3'; IGF-1R: Sense 5'-CGCAGGATGGCTATCTGTTC-3', antisense 5'-ATCACCACCGCACACTTC-3; GAPDH: Sense 5'-GTCGGTGTGAACGGATTTG-3', antisense 5'-TCCCAT TCTCAGCCTTGAC-3'. The PCR amplification cycling conditions were as follows: $95^{\circ} \mathrm{C}$ for $10 \mathrm{~min}$, followed by 40 cycles of $95^{\circ} \mathrm{C}$ for $15 \mathrm{sec}, 60^{\circ} \mathrm{C}$ for $1 \mathrm{~min}$ and $72^{\circ} \mathrm{C}$ for $1 \mathrm{~min}$.

The gene-specific primers used in RAW364.7 murine macrophages were as follows. TLR4: Sense 5'-CTATGAACA AAGGGTCTATCAG-3', antisense 5'-AAGAACAGCAAC CACTAAAG-3'; MyD88: Sense 5'-CACTCGCAGTTTGTT GGATG-3', antisense 5'-TGTAAAGGCTTCTCGGACTC-3'; PI3K: Sense 5'-ATGCCAGAAAGGAGAATG-3', antisense 5'-TGT TGGACTCAGCAATAC-3'; Akt: Sense 5'-GGG CACATCAAGATAACG-3', antisense 5'-TGGTCCTGG TTGTAGAAG-3'; NF- $\kappa B p 65$ : Sense 5'-CCCGAAACTCAA CTTCTG-3'; antisense 5'-ATCTGCCCTGATGGTAAC-3'; p38MAPK: Sense 5'-GTGTTCACACCCGCAAGGTC-3', antisense 5'-CGGTCAGCTTCTGGCACTTC-3'; GAPDH: Sense 5'-ATCACTGCCACCCAGAAG-3', antisense 5'-TCC
ACGACGGACACATTG-3'. The PCR amplification cycling conditions were as follows: $95^{\circ} \mathrm{C}$ for $10 \mathrm{~min}$, followed by 40 cycles of $95^{\circ} \mathrm{C}$ for $15 \mathrm{sec}$ and $60^{\circ} \mathrm{C}$ for $45 \mathrm{sec}$. All reactions were performed in triplicate. Relative gene expression data were determined by the $2^{-\Delta \Delta \mathrm{Cq}}$ method.

Western blotting. Western blot analysis was used to investigate Akt activation in pancreatic tissue and RAW264.7 cells. Protein samples were prepared using PRO-PREP protein extraction solution for total fractions according to the manufacturer's instructions. Total protein was separated with sodium dodecyl sulfate-polyacrylamide gel electrophoresis and transferred to polyvinylidene fluoride membranes. The membranes were blocked with $5 \%$ nonfat milk for $1 \mathrm{~h}$, incubated overnight at $4^{\circ} \mathrm{C}$ with Phospho-Akt antibody $(1: 1,000$; Cell Signaling Technology, Inc., Danvers, MA, USA) or Akt antibody (1:1,000; Cell Signaling Technology, Inc.) and then incubated with the appropriate secondary antibodies for $1 \mathrm{~h}$. GAPDH (1:10,000; Abcam, Cambridge, MA, USA) was used as an internal control. The ECL Plus kit from Amersham; (GE Healthcare Life Sciences, Chalfont, UK) was used for chemiluminescent detection. Densitometric analyses were conducted using Optimus software (Optimus Corp, Fort Collins, CO, USA) for quantitation.

Statistical analysis. Results are presented as means \pm standard deviation. All data were analyzed by one-way ANOVA tests with LSD and SNK post hoc tests using SPSS 13.0 (IBM Corp., Armonk, NY, USA). $\mathrm{P}<0.05$ was considered to indicate a statistically significant difference.

\section{Results}

Histological examination of pancreatic tissue. Histological examination found no difference between the normal and SO groups. Compared with the SO group, pancreatic injury in SAP rats was characterized by tissue edema, leukocyte infiltration and acinar cell necrosis, and the hemorrhage pathological scores for pancreatic tissues were markedly increased (Fig. 1). Treatment with IGF-1 obviously ameliorated the severity of the inflammatory response in SAP animals, alleviating tissue edema, leukocyte infiltration and acinar cell necrosis, decreasing the hemorrhage pathological scores for pancreatic injury. As the IGF-1 dosage increased, pancreatic injury was lessened and the pathological scores significantly declined, as shown in Fig. 1.

Ascites volume and ascites amylase activity. Ascites volume gradually increased after the exposure to LPS in the SAP group. The volume of ascites was significantly reduced by treatment with IGF-1, but not in a dose-dependent manner. Ascites amylase activity from the normal rats is shown in Fig. 2. In agreement with the histological changes, amylase activity in the SAP rats significantly increased compared with the SO group. The IGF-1 treatment groups had markedly decreased levels of ascites amylase compared with the SAP rats, and as the IGF-1 dosage increased the levels of amylase activity declined substantially (Fig. 2).

Inflammatory cytokine levels in serum. The measurement of serum inflammatory cytokine levels showed that SAP rats had 
A

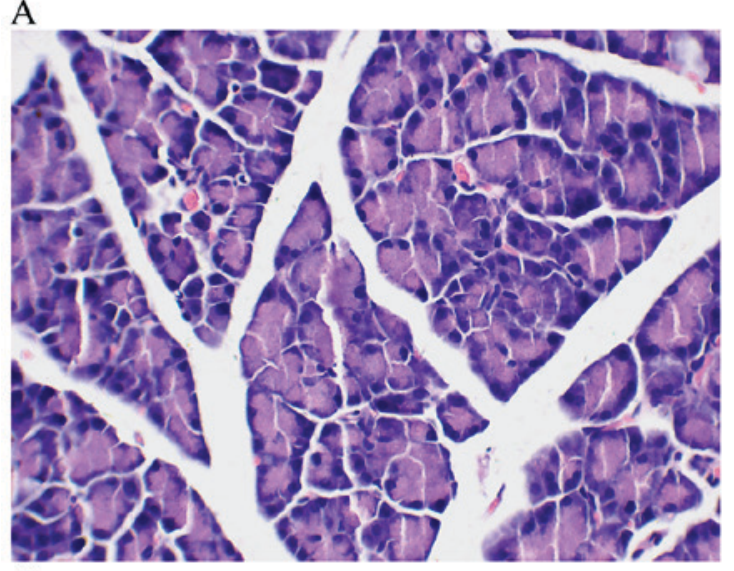

$\mathrm{C}$

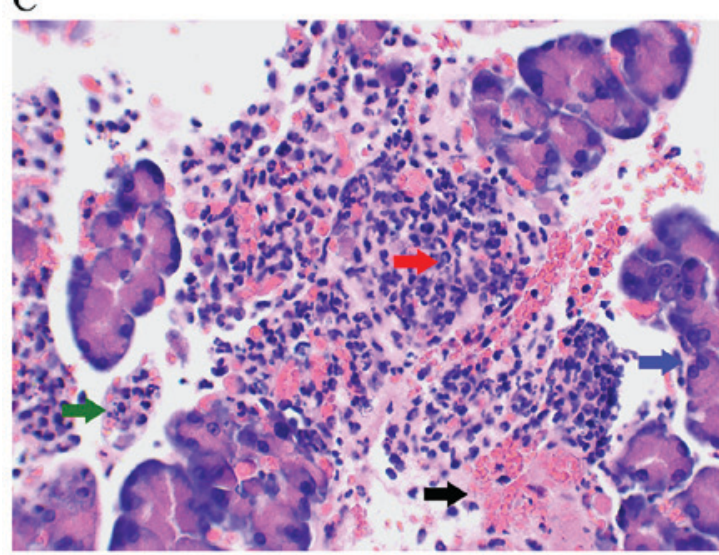

$\mathrm{E}$

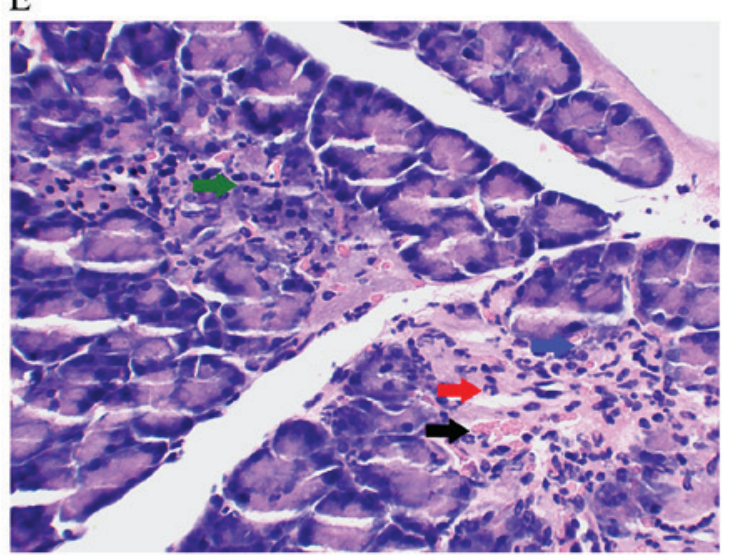

B

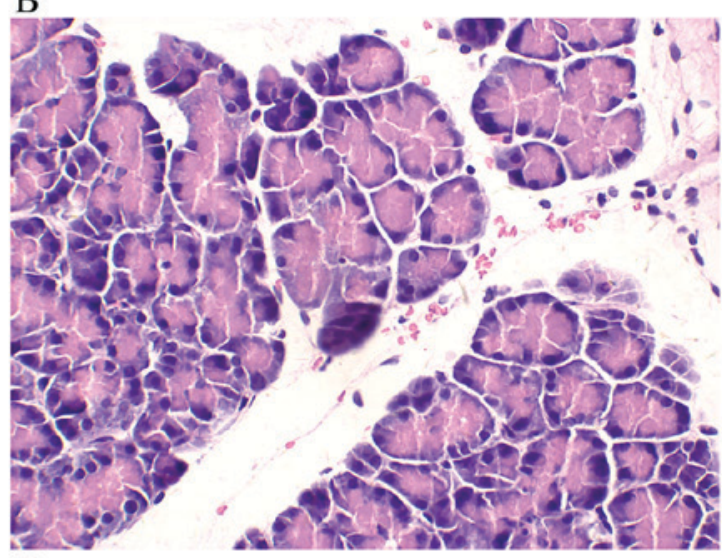

$\mathrm{D}$

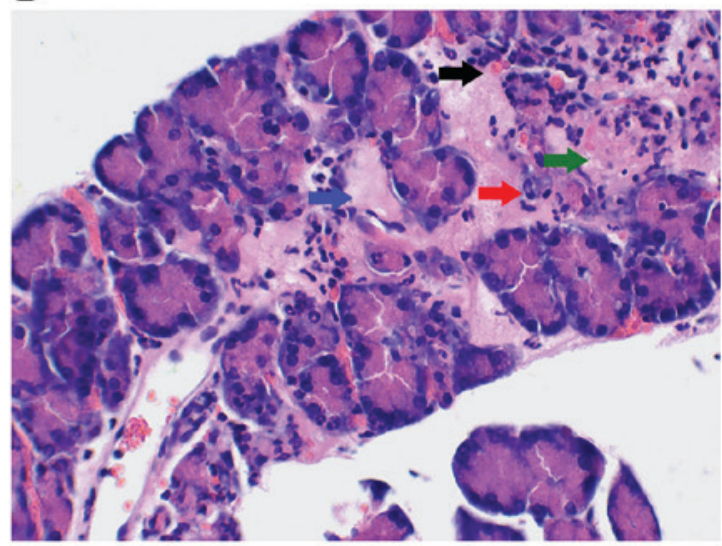

$\mathrm{F}$

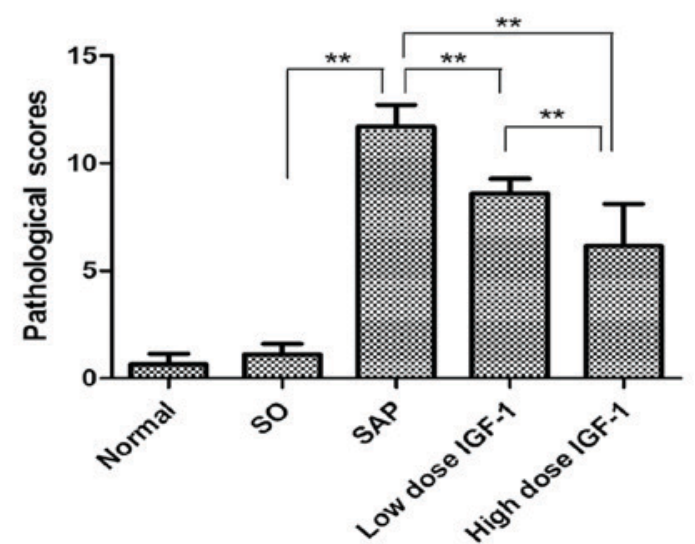

Figure 1. Histological examination of the pancreas and pathological scores for pancreatic tissue in each group at the $6 \mathrm{~h}$ time point (original magnification, 200x). (A) Normal group. (B) SO group. (C) SAP group. (D) High-dose IGF-1 group. (E) Low-dose IGF-1 group. (F) Pathological scores for pancreatic tissue. Pancreatic injury in SAP rats was characterized by tissue edema (blue arrow), leukocyte infiltration (red arrow), hemorrhage (black arrow) and acinar cell necrosis (green arrow). Treatment with IGF-1 ameliorated the severity of the inflammatory response in pancreata of SAP rats. ${ }^{* *} \mathrm{P}<0.01$. SO, sham operation; SAP, severe acute pancreatitis; IGF-1, insulin-like growth factor-1.

increased expression of the proinflammatory cytokine IL-6 compared with the SO group. In contrast, the level of IL-6 was reduced by treatment with IGF-1 in a dose-dependent manner (Fig. 2). The results indicated that IGF-1 could effectively attenuate pancreatic injury.

Effect of IGF-1 on TLR4, p38MAPK, NF-кBp65 and IGF-1R $m R N A$ levels in pancreatic tissue. The levels of TLR4, p38MAPK, NF- $\kappa$ Bp65 and IGF-1R mRNA in the five groups were assessed (Fig. 3). There was no significant difference between the normal and SO group. Compared with the SO group, TLR4, p38MAPK and NF- $\kappa$ Bp65 mRNA markedly increased in pancreatic tissues of SAP rats $(\mathrm{P}<0.05)$. High and low dose IGF-1 treatment decreased the expression of TLR4, p38MAPK and NF- $\kappa$ Bp65 mRNA in the pancreas observably. Because TLR4, NF- $\kappa$ Bp65 and p38MAPK are important signaling molecules in the inflammatory reaction associated with SAP, these results suggested that IGF-1 attenuated the sodium taurocholate-induced inflammation in the pancreas.

IGF-1R, a member of the family of transmembrane tyrosine kinases, binds IGF-1 with high affinity and initiates the physiological response to this ligand (16). Compared with the normal 

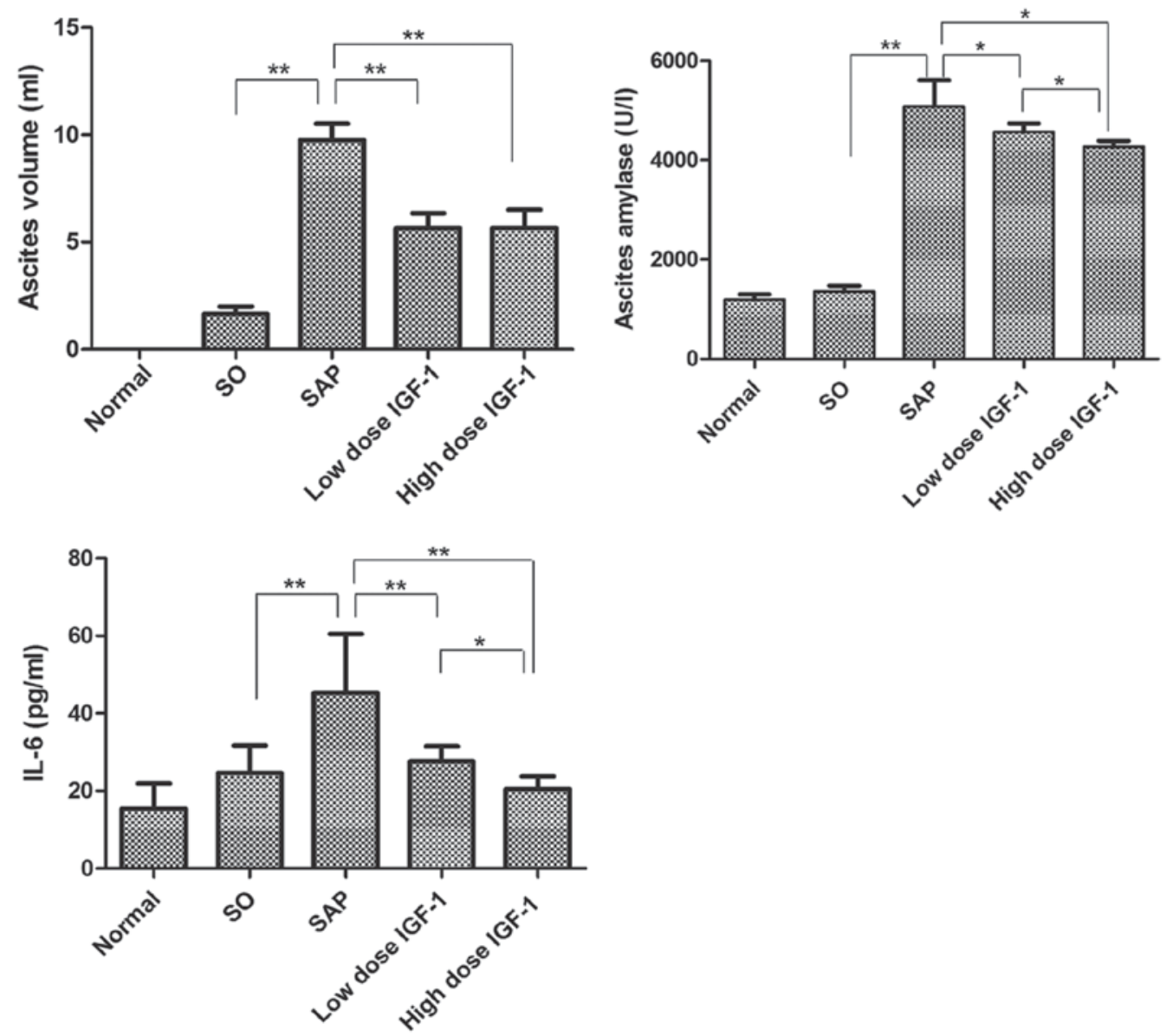

Figure 2. Ascites volume, ascites amylase activity and IL-6 levels in each group. Ascites volume, ascites amylase activity and IL-6 levels increased after exposure to LPS in the SAP group. The IGF-1 treatment group had markedly decreased ascites volume, ascites amylase activity and IL-6 expression compared with the SAP rats. ${ }^{*} \mathrm{P}<0.05 ;{ }^{* *} \mathrm{P}<0.01$. IL, interleukin; SO, sham operation; SAP, severe acute pancreatitis; IGF-1, insulin-like growth factor-1.

and SO groups, the level of IGF-1R mRNA expression fell in pancreatic tissues of SAP rats, and was significantly decreased compared with the normal group $(\mathrm{P}<0.05)$. In contrast, high and low dose IGF-1 treatment produced similar significant increases in expression of IGF-1R mRNA in the pancreas $(\mathrm{P}<0.05)$. These results suggested that exogenous IGF-1 increased the expression of IGF-1R in the same manner as endogenous IGF-1.

Effect of IGF-1 on phosphorylation of Akt in pancreatic tissue. Akt is the downstream signaling molecule of PI3K in inflammation. The phosphorylation of Akt in the SAP group was markedly upregulated compared to the SO group. Moreover, IGF-1 treatment resulted in increased protein expression of p-Akt/Akt in the pancreas (Fig. 4). These results suggested that IGF-1 could increase the phosphorylation of Akt, consistent with the results of another study (17).

Assay for cellular viability. Increasing concentrations of LPS, wortmannin and IGF-1 had no significant effect on the proliferative activity or viability of RAW264.7 cells compared with Blank cells after $6 \mathrm{~h}$ of treatment. The final concentration of LPS, IGF-1 and wortmannin was therefore set at $1 \mathrm{~g} / \mathrm{ml}, 100 \mathrm{ng} / \mathrm{ml}$ and $100 \mathrm{nM}$, respectively, for the next set of experiments.

Effect of IGF-1 and wortmannin on TNF- $\alpha$ and IL- 6 levels in RAW264.7 cells. Since macrophages play prominent roles in various inflammatory conditions, especially in SAP (18), we analyzed whether IGF-1 and wortmannin influenced the production of proinflammatory cytokines in response to the TLR4 ligand LPS in RAW264.7 macrophages (Fig. 5). The protein levels of TNF- $\alpha$ and IL- 6 were higher in the LPS group than in the Blank group $(\mathrm{P}<0.01)$. Compared with the LPS group, the levels of these cytokines were increased in the IGF-1 group, but not different from each other in statistics $(\mathrm{P}>0.05)$. We added IGF-1+wortmannin group and compared it with IGF-1 group, we found that the expression of IL- 6 and TNF- $\alpha$ were different from each other in statistics $(\mathrm{P}<0.05$; Fig. 5). Meanwhile, the level of TNF- $\alpha$ and IL- 6 were significantly decreased in the wortmannin group $(\mathrm{P}<0.05)$. These data demonstrated that agonists and inhibitors of PI3K/Akt were able to affect LPS-induced production of proinflammatory cytokines in RAW264.7 cells.

Effect of IGF-1 and wortmannin on PI3K, Akt, TLR4,

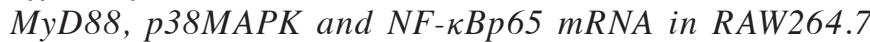
cells. In response to LPS, overexpression of TLR4, MyD88, PI3K, Akt, p38MAPK and NF- $\kappa$ Bp65 mRNA was observed in RAW264.7 cells compared with Blank cells. As shown in Fig. 6, the level of each of these factors was higher in the IGF-1 group compared with the LPS group, whereas their expression in the wortmannin group was lower than in the LPS 

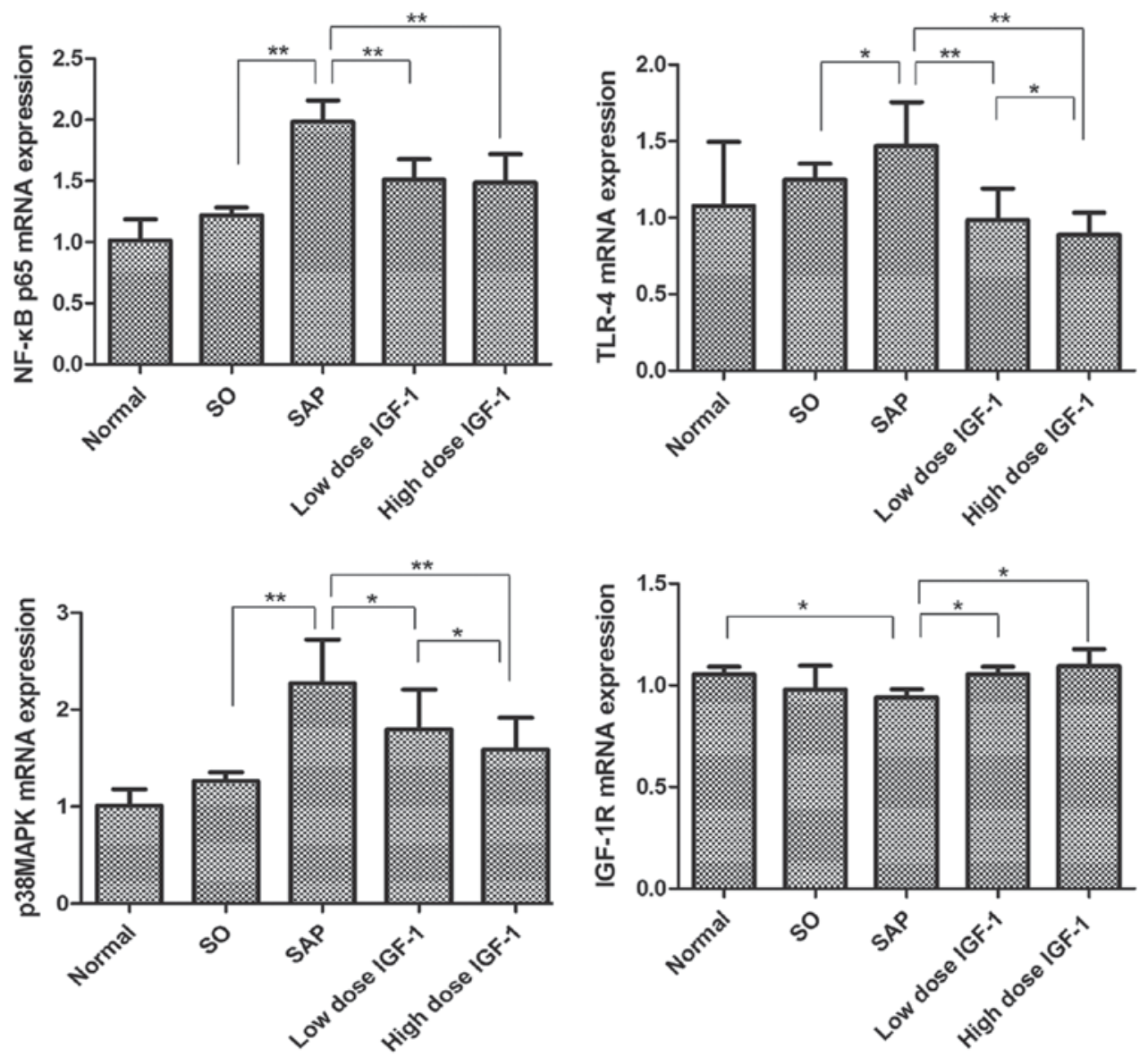

Figure 3. TLR4, p38MAPK, NF-кBp65 and IGF-1R mRNA expression in each group. " $\mathrm{P}<0.05$; ${ }^{* *} \mathrm{P}<0.01$. TLR4, Toll-like receptor 4; SO, sham operation; SAP, severe acute pancreatitis; IGF-1, insulin-like growth factor-1.

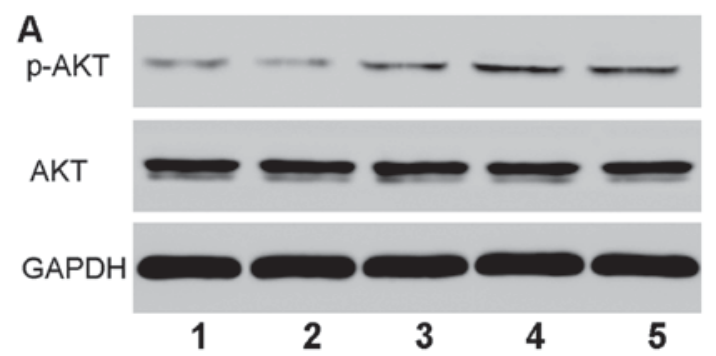

B Quantitative date

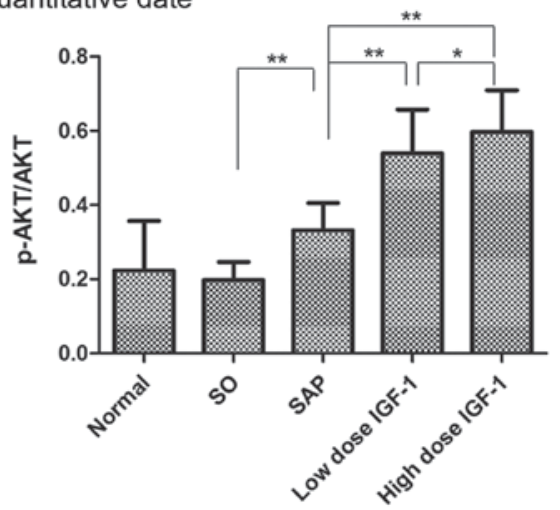

Figure 4. p-Akt/Akt protein expression in each group of rats. (A) Western blot analysis of each group. 1, Normal group; 2, SO group; 3, SAP group; 4, low-dose IGF-1 group; and 5, high-dose IGF-1 group. (B) p-Akt/Akt protein expression in each group. ${ }^{*} \mathrm{P}<0.05 ;{ }^{* *} \mathrm{P}<0.01$. SO, sham operation; SAP, severe acute pancreatitis; IGF-1, insulin-like growth factor-1. group. Moreover, the mRNA level of TLR4, MyD88, PI3K, p38MAPK and NF- $\mathrm{kBp} 65$ in the IGF-1+wortmannin group was lower than in the IGF-1 group $(\mathrm{P}<0.05)$. While the mRNA level of Akt in the IGF-1+wortmannin group was lower than in the IGF-1 group, the difference was not statistically significant (Fig. 6). These data demonstrated that agonists and inhibitors of PI3K/Akt were able to change the level of PI3K and Akt and affect gene expression of TLR4, MyD88, p38MAPK and NF-кBp65 in RAW264.7 cells.

Effect of IGF-1 on phosphorylation of Akt in RAW264.7 cells. The phosphorylation of Akt in the LPS group was markedly upregulated compared to the Blank cells (Fig. 7). Moreover, IGF-1 treatment resulted in increased protein expression of p-Akt/Akt in the cells. The phosphorylation of Akt in the wortmannin group was lower than in the LPS group, and was also lower in the IGF-1+wortmannin group compared to the IGF-1 group $(\mathrm{P}<0.05$; Fig. 7). These results suggested that IGF-1 could increase the phosphorylation of Akt in RAW264.7 cells.

\section{Discussion}

The PI3K/Akt pathway has been shown to control a variety of cellular processes, including cell survival and proliferation. Several lines of evidence have recently shown that PI3K/Akt are involved in several inflammatory diseases $(19,20)$. We have 

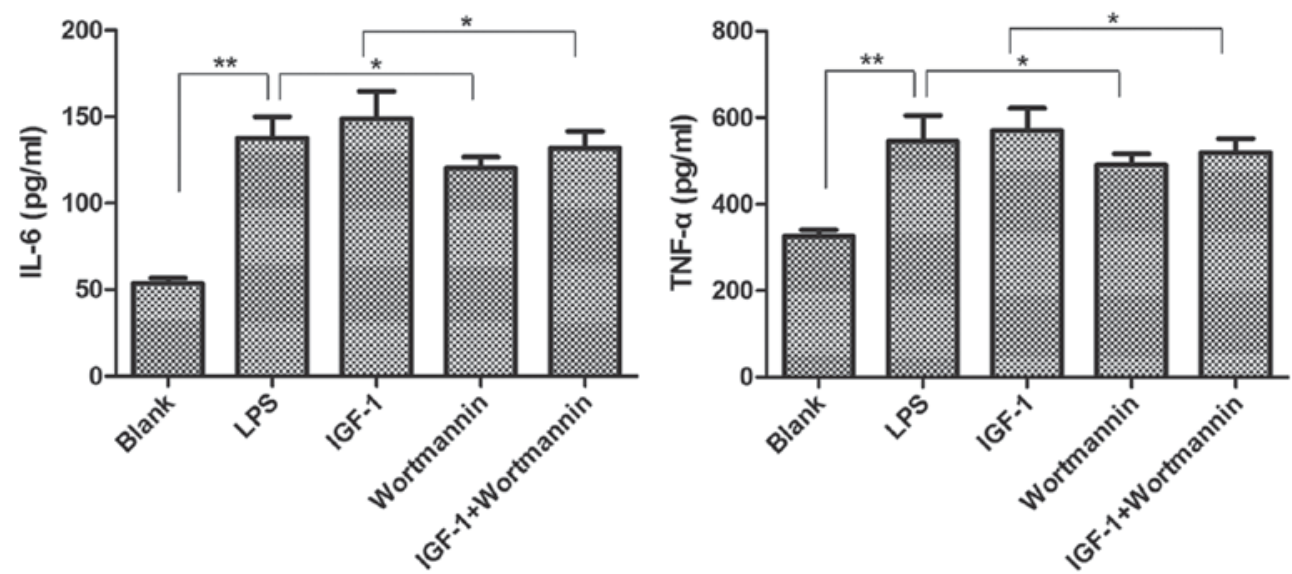

Figure 5. The level of TNF- $\alpha$ and IL-6 in each group of RAW264.7 cells. ${ }^{*} \mathrm{P}<0.05 ;{ }^{* *} \mathrm{P}<0.01$. LPS, lipopolysaccharide; IGF-1, insulin-like growth factor-1.
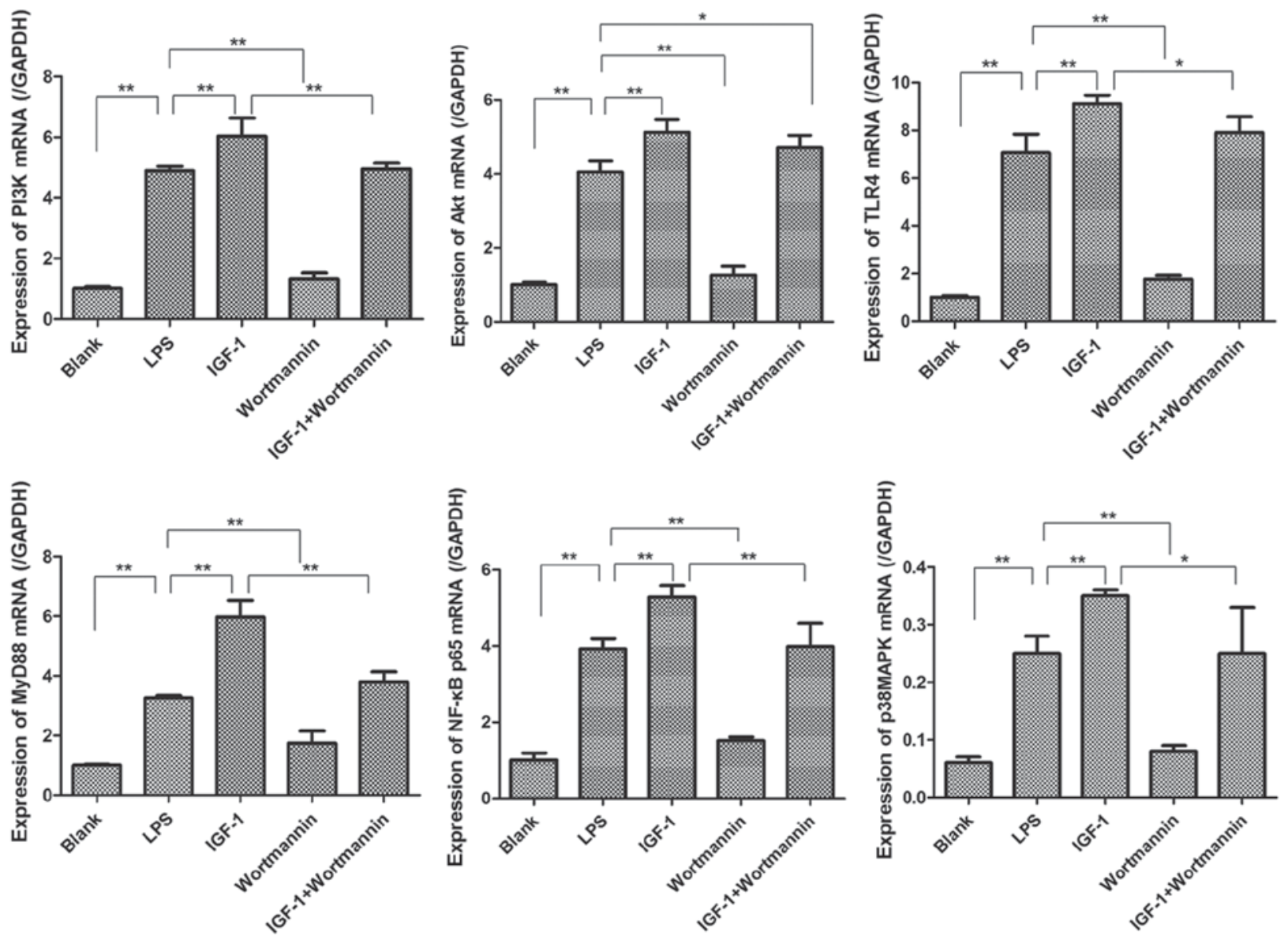

Figure 6. PI3K, Akt, TLR4, MyD88, NF-kBp65 and p38MAPK mRNA expression in each group. " $\mathrm{P}<0.05$; "* $\mathrm{P}<0.01$. PI3K, phosphoinositide 3-kinase; Akt, protein kinase B; TLR4, Toll-like receptor 4; LPS, lipopolysaccharide; IGF-1, insulin-like growth factor-1.

shown previously that the PI3K/Akt inhibitor wortmannin decreased inflammatory cytokines, alleviated the severity of inflammation in the pancreas and improved the survival rate in SAP rats, indicating that PI3K/Akt take part in the development of SAP (2). In the present study, we used IGF-1, an agonist of PI3K, to test the hypothesis that PI3K/Akt regulate proinflammatory cytokines and inflammation through TLR4 and its downstream signaling pathway, thereby controlling SAP inflammation.
We examined the severity of inflammation in SAP rats by histological examination of the pancreas, ascites volume, activity of ascites amylase and TNF- $\alpha$ level following IGF-1 treatment. The results showed that IGF-1 not only decreased the severity of pancreatic damage but also greatly attenuated the expression of the proinflammatory cytokine IL-6 in a dose-dependent manner, which was consistent with the histological changes we observed in the pancreas, and suggested that IGF-1 exerts an anti-inflammatory effect in SAP rats by 


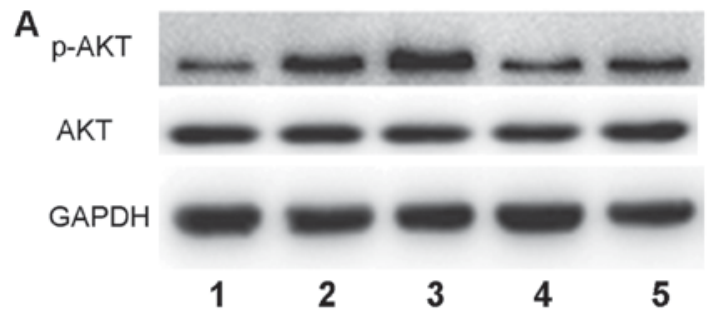

B Quantitative date

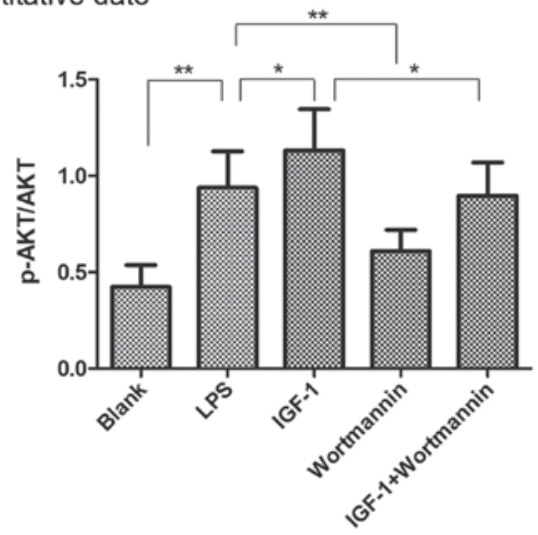

Figure 7. p-Akt/Akt protein expression in each group of RAW264.7 cells. (A) Western blot analysis of each group. 1, Blank group; 2, LPS group; 3, IGF-1 group; 4, Wortmannin group; and 5, IGF-1+wortmannin group. (B) $\mathrm{p}-\mathrm{Akt} / \mathrm{Akt}$ protein expression in each group. ${ }^{*} \mathrm{P}<0.05 ;{ }^{* *} \mathrm{P}<0.01$. LPS, lipopolysaccharide; IGF-1, insulin-like growth factor-1.

reducing the expression of proinflammatory cytokines. To determine if the modulating effect of IGF-1 on inflammation was associated with TLR4 gene expression, TLR4 mRNA levels were determined by real-time PCR. The results showed that IGF-1 decreased TLR4 expression in a dose-dependent manner. It is well known that TLR4-mediated signaling activates NF- $\mathrm{BB}$ and $\mathrm{p} 38 \mathrm{MAPK}$, which play critical roles in regulating expression of proinflammatory genes. Thus, we examined the expression of NF-кBp65 and p38MAPK and found that they changed in accordance with expression of TLR4. All of these results indicate that IGF-1 may exert a modulating effect on TLR4 and its downstream signaling pathway in SAP rats. However, there are some caveats regarding this conclusion. First, IGF-1 as an agonist of PI3K showed the same anti-inflammatory effect as the PI3K inhibitor wortmannin. Second, inhibitors were not used to inhibit the effect of IGF-1 and TLR4 in our SAP rats. Thus, it was difficult to prove that the anti-inflammatory effect of IGF-1 was mediated by TLR4 and its downstream signaling pathway. Additional study will be required to unambiguously demonstrate this effect.

To determine the relationship between PI3K/Akt and TLR4, RAW264.7 cells were treated with LPS in the presence or absence of a PI3K inhibitor (wortmannin) and agonist (IGF-1). Stimulation of RAW264.7 cells with LPS induces the production of various proinflammatory cytokines. Our data showed that IL-6 and TNF- $\alpha$ secretion were increased by co-stimulation with LPS and IGF-1 compared to LPS stimulation alone. This modulation was suppressed by using wortmannin, indicating that IGF-1 promotes the inflammatory effect in RAW264.7 cells through PI3K/Akt. Furthermore, the expression of TLR4 and MyD88 mRNA, which was increased by IGF-1 overexpression following LPS treatment, was downregulated by wortmannin. Moreover, the expression of Akt, NF- $\kappa B p 65$ and p38MAPK were in line with the expression of TLR4 and proinflammatory cytokines. It has been shown that TLR4 activation by LPS triggers the activity of MyD88, changes a series of adapter molecules and kinases and then stimulates NF-KB and p38MAPK translocation and expression of target genes (9). These findings suggest that PI3K/Akt have a relationship with TLR4 in which upstream and downstream TLR4 signaling can be affected by inhibitors and agonists of PI3K in RAW264.7 cells.

There are many TLRs. The expressions of TLR2, TLR4 and TLR9 were found to be increased in experimental AP in rodents, and there is strong data indicating that TLR4 plays a significant pro-inflammatory role in the progression of AP apart from TLR2 and TLR9 (5-7). Activation of TLR4 signaling is involved in the injury of the pancreas, intestine, lung, liver and kidney in SAP; meanwhile, expression of TLR4 was increased in the early phase of SAP in those organs $(5-7,21,22)$. TLR4 (-/-) mice showed significantly less SAP and reduced lung injury compared to wild-type mice after stimulation in the SAP animal model (5). Furthermore, another study found that TLR4 but not TLR 2 regulates inflammation and tissue damage in AP (23). TLR4 may trigger inflammatory responses through a series of signal transduction cascades, including MYD88. Knockdown of MYD88 attenuates LPS-induced inflammatory responses in pancreatic ductal cells (24). Meanwhile, acute pancreatitis patients have high level of TLR4 and decreasing the expression of TLR4 reduces the risk of systemic complications and mortality (25). NF- $\mathrm{kB}$ and p38MAPK are the two main signaling pathways activated by TLR4-mediated signaling, and both play an important role in the regulation of several inflammatory cytokines and mediators. Previous work from our group has shown that the activation of $\mathrm{NF}-\mathrm{kB}$ and p38MAPK is a prominent response during pancreatic injury and that activation of these molecules plays a key role not only in the release of inflammatory factors, but also in increasing neutrophil infiltration of the pancreas $(14,26)$. All the above research thus suggests that TLR4 plays an important role in the synthesis and release of proinflammatory cytokines, and indicates that upregulation of the TLR4 gene may be related to the development of inflammation in SAP. Our present study is consistent with those previous results.

Both in vitro and in vivo data in our present study suggested that inhibitors and agonists of PI3K could regulate the expression of TLR4 and its downstream molecules (MyD88, NF- $\mathrm{kB}$, p38MAPK, IL-6 and TNF- $\alpha$ ), indicating a relationship between PI3K and TLR4 in the progression of SAP. In fact, several reports have shown just such a relationship in other cells (27-32), as well as hypoxic stress, not only in acute pancreatitis. One study demonstrated that treatment with a PI3K inhibitor could significantly decrease LPS-induced TLR4 protein and mRNA expression in vascular smooth muscle cells (27). Other studies have shown that activation of the PI3K pathway could increase TLR4-induced TNF- $\alpha$ and IL- 6 production in mast cells (28). It has also been demonstrated that PI3K/Akt contribute to increased expression of TLR4 in macrophages exposed to hypoxic stress (29). Meanwhile, several investigators have determined that PI3K/Akt are important mediators involved in regulating NF- $\mathrm{kB}$ and p38MAPK-dependent gene expression 
in various cell types $(30,31)$. In addition, PI3K has been shown to positively regulate cytokine expression through the formation of a complex between the p85 regulatory subunit, TLR4 and MyD88, which limits LPS-induced inflammation via inhibition of TLR4/MyD88/PI3K complex formation that in turn inhibits activation of downstream signaling pathways (32). Our previous data showed that an inhibitor of PI3K (wortmannin) alleviated the severity of SAP by suppressing phosphorylation of Akt, which inhibited the activation of $N F-\kappa B$ and p38MAPK (2). In the present study, the downregulation of TLR4 and amelioration of inflammation were accompanied by a decline in the level of Akt in SAP rats. Meanwhile, our in vitro experiments produced similar results, in which inhibition of PI3K attenuated the expression of Akt, TLR4, MyD88, NF- $\kappa$ Bp65 and p38MAPK and downregulated the level of proinflammatory cytokines; in contrast, activation of PI3K with IGF-1 produced the opposite result, and wortmannin restrained the proinflammatory effect of IGF-1. In agreement with previous reports from other laboratories, we have uncovered the relationship between PI3K/Akt and TLR4, and our results provide evidence for the first time to our knowledge that PI3K/Akt may control inflammation through regulation of TLR4 and its downstream signaling in the progression of SAP.

However, there was a very mixed reaction to the application of IGF-1 in vivo and vitro. IGF-1, a peptide hormone, is synthesized in the liver and kidney and has pleiotropic effects on cell growth and metabolism (33). IGF-1R binds IGF-1 with high affinity and initiates the physiological response to this ligand, such as induce strong activation of the PI3K pathway (28). Some reports have shown that IGF-1 has an anti-inflammatory effect, and one report determined that IGF-1 alleviates ox-LDL-induced inflammation (34). In our study we employed IGF-1 as an agonist of PI3K in our study and testd the level of IGF-1R. We found that IGF-1 increased the phosphorylation of Akt, consistent with the results of other studies (17). However, IGF-1 activated RAW264.7 cells in vitro, but failed to do so in vivo. Those results demonstrated that IGF-1 can protect SAP rats in other ways than by IGF-1R in pancreatic tissue. Study reported that administration of IGF-1 attenuates pancreatic damage in part due to the increase in IL-10 production, the reduction in liberation of IL-1 $\beta$ and the improvement in pancreatic blood flow (35). Moreover, insulin protects pancreatic acinar cells from cytosolic calcium overload via inhibition of the plasma membrane calcium pump (36). Thus, the expression of IGF-1R mRNA only reflect the effect of exogenous IGF-1. Further in-depth studies will be necessary to interpret the discrepancy between IGF-1 in vivo and in vitro effects.

Taken together, our results suggest that PI3K/Akt may regulate TLR4 and its downstream adapter molecules, kinases, transcription factors and expression of inflammatory cytokinse, thereby controlling SAP inflammation. Akt and TLR4 knockdown experiment would be done to futher confirm this conculsion in the future. We also found that IGF-1 could inhibit inflammation in SAP rats. Based on our previous and present studies, we suggest that pharmacologic inhibition of the PI3K/Akt pathway may represent a new therapeutic approach to limit the progress and development of inflammation in SAP. It is also important to note that because signaling pathways in inflammation are linked together in an interrelated network, further studies will be required to accurately investigate the mechanisms of PI3K/Akt and TLR4 in the progression of SAP.

\section{Acknowledgements}

Not applicable.

\section{Funding}

This study was supported by Medical leading professional project of Songjiang district, Shanghai Health Bureau (grant no. 201358) and Shanghai Health Bureau Youth Scientific Research Project. (grant no. 20144Y0161).

\section{Availability of data and materials}

The datasets used and analyzed during the current study are available from the corresponding author on reasonable request.

\section{Authors' contributions}

JW conceived and performed the experiments and was a major contributor in writing the manuscript. PX designed the experiments and analyzed the cell data. CZ and ZWY analyzed and interpreted the data regarding the animal studies and performed polymerase chain reaction and western blot analysis of the pancreases and cells. CZW and YXL performed the histological examination of the pancreases. All authors read and approved the final manuscript.

\section{Ethics approval and consent to participate}

The experiment was designed in accordance with the guidelines for the care and use of laboratory animals in research and was approved by the Ethical and Research Committee of Shanghai Jiao Tong University.

\section{Consent for publication}

Not applicable.

\section{Competing interests}

The authors declare that they have no competing interests.

\section{References}

1. Chiang DT, Anozie A, Fleming WR and Kiroff GK: Comparative study on acute pancreatitis management. ANZ J Surg 74: 218-221, 2004.

2. Xu P, Wang J, Yang ZW, Lou XL and Chen C: Regulatory roles of the PI3K/Akt signaling pathway in rats with severe acute pancreatitis. PLoS One 8: e81767, 2013.

3. Troutman TD, Hu W, Fulenchek S, Yamazaki T, Kurosaki T, Bazan JF and Pasare C: Role for B-cell adapter for PI3K (BCAP) as a signaling adapter linking Toll-like receptors (TLRs) to serine/threonine kinases PI3K/Akt. Proc Natl Acad Sci USA 109: 273-278, 2012.

4. Akira S, Uematsu S and Takeuchi O: Pathogen recognition and innate immunity. Cell 124: 783-801, 2006.

5. Sharif R, Dawra R, Wasiluk K, Phillips P, Dudeja V, Kurt-Jones E, Finberg R and Saluja A: Impact of toll-like receptor 4 on the severity of acute pancreatitis and pancreatitis-associated lung injury in mice. Gut 58: 813-819, 2009. 
6. Ding JL, Zhou ZG, Zhou XY, Zhou B, Wang L, Wang R, Zhan L, Sun XF and Li Y: Attenuation of acute pancreatitis by peroxisome proliferator-activated receptor- $\alpha$ in rats: The effect on toll-like receptor signaling pathways. Pancreas 42: 114-122, 2013.

7. Hoque R, Sohail M, Malik A, Sarwar S, Luo Y, Shah A, Barrat F, Flavell R, Gorelick F, Husain S and Mehal W: TLR9 and the NLRP3 inflammasome link acinar cell death with inflammation in acute pancreatitis. Gastroenterology 141: 358-369, 2011.

8. Mogensen TH: Pathogen recognition and inflammatory signaling in innate immune defenses. Clin Microbiol Rev 22: 240-273, 2009.

9. Takeda $\mathrm{K}$ and Akira S: TLR signaling pathways. Semin Immunol 16: 3-9, 2004.

10. Gay NJ and Gangloff M: Structure and function of Toll receptors and their ligands. Annu Rev Biochem 76: 141-165, 2007.

11. Schmid RM and Adler G: NF-kappaB/rel/IkappaB: Implications in gastrointestinal diseases. Gastroenterology 118: 1208-1228, 2000 .

12. Zarubin $\mathrm{T}$ and Han $\mathrm{J}$ : Activation and signaling of the p38 MAP kinase pathway. Cell Res 15: 11-18, 2005.

13. Xu P, Zhou XJ, Chen LQ, Chen J, Xie Y, Lv LH and Hou XH: Pioglitazone attenuates the severity of sodium taurocholate-induced severe acute pancreatitis. World J Gastroenterol 13 : 1983-1988, 2007.

14. Wang J, Xu P, Hou YQ, Xu K, Li QH and Huang L: Pancreatitis-associated ascitic fluid induces proinflammatory cytokine expression in THP-1 cells by inhibiting anti-inflammatory signaling. Pancreas 42: 855-860, 2013.

15. Schmidt J, Rattner DW, Lewandrowski K, Compton CC, Mandavilli U, Knoefel WT and Warshaw AL: A better model of acute pancreatitis for evaluating therapy. Ann Surg 215: 44-56, 1992.

16. LeRoith D, Werner H, Beitner-Johnson D and Roberts CT Jr: Molecular and cellular aspects of the insulin-like growth factor I receptor. Endocr Rev 16: 143-163, 1995.

17. Zhang L, Yue Y, Ouyang M, Liu H and Li Z: The effects of IGF-1 on TNF- $\alpha$-treated DRG neurons by modulating ATF3 and GAP-43 expression via PI3K/Akt/S6K signaling pathway. Neurochem Res 42: 1403-1421, 2017.

18. Metz M and Maurer M: Mast cells-key effector cells in immune responses. Trends Immunol 28: 234-241, 2007.

19. Konrad S, Ali SR, Wiege K, Syed SN, Engling L, Piekorz RP, Hirsch E, Nürnberg B, Schmidt RE and Gessner JE: Phosphoinositide 3-kinases gamma and delta, linkers of coordinate $\mathrm{C} 5$ a receptor-Fcgamma receptor activation and immune complex-induced inflammation. J Biol Chem 283: 33296-33303, 2008.

20. González-García A, Sánchez-Ruiz J, Flores JM and Carrera AC: Phosphatidylinositol 3-kinase gamma inhibition ameliorates inflammation and tumor growth in a model of colitis-associated cancer. Gastroenterology 138: 1374-1383, 2010.

21. Sawa H, Ueda T, Takeyama Y, Yasuda T, Shinzeki M, Matsumura N, Nakajima T and Kuroda Y: Expression of toll-like receptor 2 and 4 in intestinal mucosa in experimental severe acute pancreatitis. Hepatogastroenterology 55: 2247-2251, 2008.

22. Zhang X, Zhu C, Wu D and Jiang X: Possible role of toll-like receptor 4 in acute pancreatitis. Pancreas 39: 819-824, 2010.

23. Awla D, Abdulla A, Regnér S and Thorlacius H: TLR4 but not TLR2 regulates inflammation and tissue damage in acute pancreatitis induced by retrograde infusion of taurocholate. Inflamm Res 60: 1093-1098, 2011.
24. Liu Y, Li Y, Chen KL, Zhou B, Lv ZY, Zhou ZG and Li Y: Knockdown of myeloid differentiation factor 88 attenuates lipopolysaccharide-induced inflammatory response in pancreatic ductal cells. Pancreas 45: 755-760, 2016.

25. Gorsky VA, Agapov MA, Khoreva MV and Leonenko IV: The effect of lornoxicam on TLR2 and TLR4 messenger RNA expression and tumor necrosis factor- $\alpha$, interleukin- 6 , and interleukin-8 secretion in patients with systemic complications of acute pancreatitis. Pancreas 44: 824-830, 2015.

26. Xu P, Xu K, Wang J, Jiang JP and Chen LQ: Pioglitazone: A promising therapeutic tool in sodium taurocholate-induced severe acute pancreatitis. Dig Dis Sci 56: 1082-1089, 2011.

27. Jiang D, Li D, Cao L, Wang L, Zhu S, Xu T, Wang C and Pan D: Positive feedback regulation of proliferation in vascular smooth muscle cells stimulated by lipopolysaccharide is mediated through the TLR 4/Racl/Akt pathway. PLoS One 9: e92398, 2014.

28. Hochdörfer T, Kuhny M, Zorn CN, Hendriks RW, Vanhaesebroeck B, Bohnacker T, Krystal G and Huber M: Activation of the PI3K pathway increases TLR-induced TNF- $\alpha$ and IL- 6 but reduces IL- $1 \beta$ production in mast cells. Cell Signal 23: 866-875, 2011.

29. Kim SY, Jeong E, Joung SM and Lee JY: PI3K/Akt contributes to increased expression of Toll-like receptor 4 in macrophages exposed to hypoxic stress. Biochem Biophys Res Commun 419: 466-471, 2012

30. Steelman LS, Pohnert SC, Shelton JG, Franklin RA, Bertrand FE and McCubrey JA: JAK/STAT, Raf/MEK/ERK, PI3K/Akt and BCR-ABL in cell cycle progression and leukemogenesis. Leukemia 18: 189-218, 2004.

31. Kang X, Wang LZ, Wang YG, Liu L, Fan ZW, Bai LZ and Lu XG: Expression and significance of phosphatidylinositol 3-kinase/protein kinase B signal transduction pathway in severe acute pancreatitis-associated lung injury. Zhonghua Yi Xue Za Zhi 90: 732-737, 2010 (In Chinese).

32. Endale M, Park SC, Kim S, Kim SH, Yang Y, Cho JY and Rhee MH: Quercetin disrupts tyrosine-phosphorylated phosphatidylinositol 3-kinase and myeloid differentiation factor-88 association, and inhibits MAPK/AP-1 and IKK/NF- $\mathrm{KB}$-induced inflammatory mediators production in RAW 264.7 cells. Immunobiology 218: 1452-1467, 2013.

33. Payne JF, Tangpricha V, Cleveland J, Lynn MJ, Ray R and Srivastava SK: Serum insulin-like growth factor-I in diabetic retinopathy. Mol Vis 17: 2318-2324, 2011.

34. Yu X, Xing C, Pan Y, Ma H, Zhang J and Li W: IGF-1 alleviates ox-LDL-induced inflammation via reducing HMGB1 release in HAECs. Acta Biochim Biophys Sin (Shanghai) 44: 746-751, 2012.

35. Warzecha Z, Dembinski A, Ceranowicz P, Konturek SJ, Tomaszewska R, Stachura J and Konturek PC: IGF-1 stimulates production of interleukin-10 and inhibits development of caerulein-induced pancreatitis. J Physiol Pharmacol 54: 575-590, 2003.

36. Mankad P1, James A, Siriwardena AK, Elliott AC and Bruce JI: Insulin protects pancreatic acinar cells from cytosolic calcium overload and inhibition of plasma membrane calcium pump. J Biol Chem 287: 1823-1836, 2012. 